TRANSACTIONS OF THE

AMERICAN MATHEMATICAL SOCIETY

Volume 354, Number 7, Pages 2557-2577

S 0002-9947(02)02977-X

Article electronically published on March 11, 2002

\title{
NEW WEIGHTED ROGERS-RAMANUJAN PARTITION THEOREMS AND THEIR IMPLICATIONS
}

\author{
KRISHNASWAMI ALLADI AND ALEXANDER BERKOVICH
}

\begin{abstract}
This paper has a two-fold purpose. First, by considering a reformulation of a deep theorem of Göllnitz, we obtain a new weighted partition identity involving the Rogers-Ramanujan partitions, namely, partitions into parts differing by at least two. Consequences of this include Jacobi's celebrated triple product identity for theta functions, Sylvester's famous refinement of Euler's theorem, as well as certain weighted partition identities. Next, by studying partitions with prescribed bounds on successive ranks and replacing these with weighted Rogers-Ramanujan partitions, we obtain two new sets of theorems - a set of three theorems involving partitions into parts $\not \equiv 0, \pm i$ $(\bmod 6)$, and a set of three theorems involving partitions into parts $\not \equiv 0, \pm i$ $(\bmod 7), i=1,2,3$.
\end{abstract}

\section{INTRODUCTION}

The theory of partitions is rich in examples of identities whose combinatorial interpretation yields the equality of partition functions defined in very different ways. Recently Alladi [2], 3], has developed a theory of weighted partition identities which deals with partition functions which are unequal, but where equality is attained by attaching weights. In this paper we obtain several new results by attaching weights to Rogers-Ramanujan partitions, namely, partitions into parts differing by $\geq 2$. In all instances the weights are defined multiplicatively. In [2], 3], the results primarily deal with the case where one set of partitions is a subset of the other, and where positive integral weights are attached to the smaller set of partitions. The results in this paper are further significant examples of such weighted identities and also deal with the more general situation of two unequal sets of partitions where weights positive or negative could be attached to either set.

In the first part of the paper $(\S 1-\S 6)$, we obtain a reformulation of a deep theorem of Göllnitz [15] and discuss the implications. The new reformulation is stated as Theorem 1 in $\S 1$ and is of great interest because it has several important consequences. Special cases of Theorem 1 yield Jacobi's triple product identity for theta functions (see §2), Sylvester's famous refinement of Euler's theorem (see §3), and

Received by the editors September 1, 2001.

2000 Mathematics Subject Classification. Primary 11P83, 11P81; Secondary 05A19.

Key words and phrases. Göllnitz theorem, Rogers-Ramanujan partitions, method of weighted words, Jacobi triple product identity, Sylvester's theorem, weighted partition identities, successive ranks.

Research of the first author supported in part by the National Science Foundation Grant DMS 0088975.

Research of the second author supported in part by a University of Florida CLAS Research Award. 
a weighted partition identity connecting partitions into distinct parts and RogersRamanujan partitions (see $\S 4$ ). The proof of Theorem 1 is given in $\S 5$ and $\S 6$, with $\S 5$ describing the necessary prerequisites, namely, the method of weighted words of Alladi-Andrews-Gordon [5], and $\S 6$ giving the details of the proof.

In the second part of the paper $(\S 7-\S 11)$, we study partitions with prescribed bounds on successive ranks and convert these into Rogers-Ramanujan partitions with weights. This leads to two new sets of partition theorems - three results (Theorems 2,3 , and 4$)$ involving partitions into parts $\not \equiv 0, \pm i(\bmod 6)$, and three more (Theorems 5,6 , and 7$)$ concerning partitions into parts $\not \equiv 0, \pm i(\bmod 7)$, for $i=1,2,3$. Theorems $2-7$ are appealing because the Rogers-Ramanujan partitions which had traditionally been associated with the modulus 5 (see Andrews [9, Ch. $7]$ ), are for the first time, by means of weights, connected to partitions which are defined using congruence conditions modulo 6 and 7 ; the weights for the theorems pertaining to the modulus 6 are powers of 2 , whereas the weights for the results concerning the modulus 7 are products of Fibonacci numbers.

In $\S 7$ we describe the necessary preliminaries and historical background, namely, the work of Andrews and others [8], [10]-14]. The proof of Theorem 2 connecting weighted Rogers-Ramanujan partitions with partitions into parts $\not \equiv 0, \pm 1(\bmod 6)$ is given in full in $\S 8$. Theorem 3 dealing with partitions into parts $\not \equiv 0, \pm 2(\bmod 6)$ and Theorem 4 concerning partitions into parts $\not \equiv 0, \pm 3(\bmod 6)$ are stated in $\S 9$, but their proofs are only sketched because they are similar to the proof of Theorem 2. We draw attention here that the condition $\not \equiv 0, \pm 3(\bmod 6)$ has to be interpreted properly, and when done so, leads to a very interesting new result (Theorem 4 of $\S 9)$ involving unrestricted partitions.

In $\oint 10$ and $\S 11$ we state and prove the three new theorems connecting weighted Rogers-Ramanujan partitions with partitions into parts $\not \equiv 0, \pm i(\bmod 7), i=1,2,3$. Once again, only Theorem 5 which deals with parts $\not \equiv 0, \pm 3(\bmod 7)$ is proved in full in $\S 10$, whereas proofs of Theorems 6 and 7 dealing with parts $\not \equiv 0, \pm i(\bmod 7)$, $i=2,1$, are only sketched in $\S 11$ because the details are similar.

Finally, in $§ 12$, we describe briefly some future prospects of this research.

We conclude this section by describing some notation.

We denote the set of all Rogers-Ramanujan partitions by $\mathcal{R}$, and the subset of $\mathcal{R}$ consisting of partitions not having 1 as a part by $\mathcal{R}_{2}$. Given $\pi \in \mathcal{R}$, by a chain we mean a maximal block of parts differing by 2 . Thus every $\pi \in \mathcal{R}$ can be decomposed into chains, and the parts in a given chain are all of the same parity. For a chain $\chi$, we define its length $\ell=\ell(\chi)$ to be the number of parts in $\chi$, and $\lambda=\lambda(\chi)$ to be its least part.

For any partition $\pi$, by $\sigma(\pi)$ we mean the sum of the parts of $\pi$, and by $\nu(\pi)$, the number of parts of $\pi$. We also use the standard notation that for any complex number $a$, the symbol $(a)_{n}$ is defined by

$$
(a)_{n}=(a ; q)_{n}=\left\{\begin{array}{l}
\prod_{j=0}^{n-1}\left(1-a q^{j}\right), \text { if } n>0 \\
1, \text { if } n=0
\end{array}\right.
$$

and

$$
(a)_{\infty}=(a ; q)_{\infty}=\lim _{n \rightarrow \infty}(a)_{n} .
$$


As can be seen from the above, when the base in the product is $q$, it is often suppressed, but not when it is anything other than $q$. Further notation will be introduced when necessary.

\section{A reformulation of the Göllnitz theorem}

Given a Rogers-Ramanujan partition $\pi$, decompose it into chains. For a chain $\chi$ of length $\ell$, we define its weight $\omega(\chi)$ by

$$
\omega(\chi)=\left\{\begin{array}{l}
c^{\ell-1}(c+a b), \text { if } \lambda(\chi) \text { is even, } \\
a^{\ell}+(1+c) \sum_{k=1}^{\ell-1} a^{k} b^{\ell-k}+b^{\ell}, \text { if } \lambda(\chi)=1, \\
(1+c)\left\{a^{\ell}+(1+c) \sum_{k=1}^{\ell-1} a^{k} b^{\ell-k}+b^{\ell}\right\}, \text { if } \lambda(\chi)>1 \text { is odd, }
\end{array}\right.
$$

where $a, b$, and $c$ are free parameters whose role will be described soon. Finally, the weight $\omega(\pi)$ of a Rogers-Ramanujan partition is defined as the product of the weight of its chains $\chi$; that is,

$$
\omega(\pi)=\prod_{\chi} \omega(\chi)
$$

Next, let $\vec{\pi}=\left(\pi_{1} ; \pi_{2} ; \pi_{3}\right)$ denote a vector partition with $\pi_{1}$ and $\pi_{2}$ having distinct odd parts, and $\pi_{3}$ having distinct even parts. Denote by $\mathcal{V}$ the set of all such vector partitions.

Our goal is to prove the following result and discuss its implications.

Theorem 1. Let $\mathcal{R}, \mathcal{V}$ and $\omega$ be as above. Then for any integer $n \geq 0$,

$$
\sum_{\pi \in \mathcal{R}, \sigma(\pi)=n} \omega(\pi)=\sum_{\vec{\pi} \in \mathcal{V}, \sigma(\vec{\pi})=n} a^{\nu\left(\pi_{1}\right)} b^{\nu\left(\pi_{2}\right)} c^{\nu\left(\pi_{3}\right)} .
$$

The proof of Theorem 1 is given at the end of $\S 6$. It is based on the method of weighted words due to Alladi-Andrews-Gordon [5], which provided a refinement and generalization of a deep theorem of Göllnitz [15]. The main ideas of [5] are described in $\S 5$.

Before we give the proof of Theorem 1, we discuss its implications in the next three sections.

\section{JACOBI'S TRIPLE PRODUCT IDENTITY}

From the definition of $\mathcal{V}$ it follows that

$$
\left(-a q ; q^{2}\right)_{\infty}\left(-b q ; q^{2}\right)_{\infty}\left(-c q^{2} ; q^{2}\right)_{\infty}=\sum_{n \geq 0} \sum_{\substack{\vec{\pi} \in \mathcal{V} \\ \sigma(\vec{\pi})=n}} a^{\nu\left(\pi_{1}\right)} b^{\nu\left(\pi_{2}\right)} c^{\nu\left(\pi_{3}\right)} q^{n} .
$$

Now take

$$
a b=1 \quad \text { and } \quad c=-1 .
$$

So the product on the left in $(2.1)$ is

$$
\left(-a q ; q^{2}\right)_{\infty}\left(-a^{-1} q ; q^{2}\right)_{\infty}\left(q^{2} ; q^{2}\right)_{\infty}
$$

The choices (2.2) imply that

$$
c+a b=0 \quad \text { and } \quad 1+c=0 .
$$


Therefore for partitions $\pi \in \mathcal{R}$, the only chains with non-zero weights will be the chains

$$
\chi: 1+3+5+\ldots+(2 n-1), \quad n \geq 1,
$$

for which

$$
\omega(\chi)=a^{n}+b^{n}=a^{n}+a^{-n}, \quad n \geq 1 .
$$

Thus the only partitions $\pi \in \mathcal{R}$ which will have non-zero weights will be the partitions of $n^{2}$ given by $1+3+\ldots+(2 n-1)$ with weights $\omega(\pi)=\omega(\chi)$ as in $(2.6)$. Thus by Theorem 1 and (2.2) through (2.6) we get

$$
1+\sum_{n=1}^{\infty}\left(a^{n}+a^{-n}\right) q^{n^{2}}=\sum_{n=-\infty}^{\infty} a^{n} q^{n^{2}}=\left(-a q ; q^{2}\right)_{\infty}\left(-a^{-1} q ; q^{2}\right)_{\infty}\left(q^{2} ; q^{2}\right)_{\infty}
$$

which is Jacobi's triple product identity for theta functions.

\section{Sylvester's REFinement of Euler's THEOREM}

In an important paper of 1882, Sylvester [18 improved many partition results of Euler by exploiting combinatorial techniques. In particular, Sylvester proved:

Theorem S. Let $k$ and $n$ be positive integers. Then the number of partitions of $n$ into odd parts of which exactly $k$ are different, equals the number of partitions of $n$ into distinct parts which can be grouped into $k$ maximal blocks of consecutive integers.

Euler's famous theorem on the equality of partitions of $n$ into odd parts and distinct parts follows from Theorem S by summing over $k$.

We now show that Theorem $\mathrm{S}$ follows from Theorem 1 . To this end, take

$$
c=1, \quad a+b=0 .
$$

Then the product in (2.1) can be rewritten as

$$
\prod_{m=1}^{\infty}\left(1+a b q^{4 m-2}\right)\left(1+q^{2 m}\right)=\prod_{m=1}^{\infty}\left(\frac{1+a b q^{4 m-2}}{1-q^{4 m-2}}\right) .
$$

Note that since

$$
\frac{1+x q^{n}}{1-q^{n}}=1+(1+x)\left(q^{n}+q^{2 n}+q^{3 n}+\ldots\right),
$$

the product on the right in (3.2) has the interpretation that it is the generating function for partitions into parts $\equiv 2(\bmod 4)$ where such partitions $\pi$ are counted with weights

$$
(1+a b)^{\nu_{d}(\pi)}
$$

where $\nu_{d}(\pi)$ is the number of different parts of $\pi$.

Next observe that with $c=1$ we have

$$
a^{n}+2 \sum_{j=1}^{n-1} a^{n-j} b^{j}+b^{n}=(a+b) \sum_{j=0}^{n-1} a^{n-1-j} b^{j} .
$$


So if $(a+b)=0$, then it follows from (3.5) that all odd chains in (1.1) must have weight 0 . Thus the only chains with non-zero weights are the partitions into even parts differing by $\geq 2$. The weight of such partitions given by (1.2) will be

$$
(1+a b)^{k}
$$

where $k$ is the number of even chains. Thus from (3.4) and (3.6), Theorem S follows dilated by a factor of 2 , where the odd numbers are replaced by integers $\equiv 2(\bmod 4)$ and the positive integers are replaced by even numbers.

\section{Two Weighted PARTition theOREMS}

Take

$$
b=c=1 \quad \text { and } \quad a=0
$$

in Theorem 1. Then the product (2.1) is

$$
\prod_{m=1}^{\infty}\left(1+q^{m}\right)=\sum_{n=0}^{\infty} D(n) q^{n}
$$

the generating function of $D(n)$, the number of partitions of $n$ into distinct parts.

Next, the choices in (4.1) imply that in (1.1) all even chains have weight 1 , all odd chains $\chi$ with $\lambda(\chi)=1$ have weight 1 , and all odd chains $\chi$ with $\lambda(\chi)>1$ have weight 2 . Thus by $(1.2)$

$$
\omega(\pi)=2^{k},
$$

where $k$ is the number of odd chains $\chi$ of $\pi$ with $\lambda(\chi)>1$. So we get the following result as a consequence of Theorem 1 :

Theorem A (Alladi [2]). Let $D(n)$ denote the number of partitions of $n$ into distinct parts. Given $\pi \in \mathcal{R}$, let its weight $\omega(\pi)=2^{k}$, where $k$ is the number of odd chains $\chi$ of $\pi$ with $\lambda(\chi)>1$. Then

$$
\sum_{\substack{\pi \in \mathcal{R} \\ \sigma(\pi)=n}} \omega(\pi)=D(n)
$$

Remarks. Theorem 10 of 2 is actually the same result as Theorem A, but is stated differently. In 2, $k$ is interpreted as the number of gaps between the odd parts of $\pi$ and -1 which are $>2$. The proof of this result in [2 involves constructing a (combinatorial) surjective map between the set of partitions into distinct parts and its subset, namely the set of Rogers-Ramanujan partitions.

Since partitions into odd parts are equinumerous with partitions into distinct parts, it is interesting to ask whether there is another weighted partition identity connecting partitions into odd parts and Rogers-Ramanujan partitions. For this purpose take

$$
a=b=c=1
$$

in Theorem 1. Now rewrite the product in (2.1) as

$$
\left(-q ; q^{2}\right)_{\infty}\left(-q ; q^{2}\right)_{\infty}\left(-q^{2} ; q^{2}\right)_{\infty}=\prod_{m=1}^{\infty}\left(1+q^{2 m-1}\right)\left(1+q^{m}\right)=\prod_{m=1}^{\infty}\left(\frac{1+q^{2 m-1}}{1-q^{2 m-1}}\right) .
$$


By (3.3), the product in (4.5) has the interpretation that it is the generating function of partitions in $\pi^{\prime}$ into odd parts, where $\pi^{\prime}$ is counted with weight

$$
\omega_{1}\left(\pi^{\prime}\right)=2^{\nu_{d}\left(\pi^{\prime}\right)} .
$$

In (4.6), as in Sylvester's theorem, $\nu_{d}\left(\pi^{\prime}\right)$ is the number of different parts of $\pi^{\prime}$.

Finally, note that with the choices in (4.4), the weights in (1.1) become

$$
\omega(\chi)=\left\{\begin{array}{l}
2, \text { if } \chi \text { has even parts, } \\
2 \ell, \text { if } \ell(\chi)=\ell \text { and } \lambda(\chi)=1 \\
4 \ell, \text { if } \ell(\chi)=\ell, \text { and } \lambda(\chi)>1
\end{array}\right.
$$

With these values of $w(\chi)$, and with $w(\pi)$ defined by (1.2), we get from (4.4), (4.5), and (4.6) the following new weighted partition theorem:

Theorem B. Let $\mathcal{O}$ denote the set of partitions into odd parts. Then

$$
\sum_{\pi \in \mathcal{R}, \sigma(\pi)=n} \omega(\pi)=\sum_{\pi^{\prime} \in \mathcal{O}, \sigma\left(\pi^{\prime}\right)=n} 2^{\nu_{d}\left(\pi^{\prime}\right)} .
$$

Remarks. In $\S 8$, by replacing $D(n)$ with the number of partitions of $n$ into odd parts, and by thinking of odd parts as being in residue classes $\equiv 1,3,5(\bmod 6)$, Theorem A is reformulated and a new proof given (see Theorem 3). This has the advantage of producing two other similar results (Theorem 2 of $\S 8$ and Theorem 4 of $\S 9)$, both of which are new.

\section{The METHOD OF WEIGHTED WORDS}

In 1967, Göllnitz [15] proved the following deep partition theorem:

Theorem G. Let $B(n)$ denote the number of partitions of $n$ into distinct parts $\equiv 2,4$, or $5(\bmod 6)$.

Let $C(n)$ denote the number of partitions of $n$ in the form $m_{1}+m_{2}+\cdots+m_{\nu}$ such that $m_{\nu} \neq 1,3$, and $m_{i}-m_{i+1} \geq 6$ with strict inequality if $m_{i} \equiv 0,1$, or $3(\bmod 6)$. Then

$$
B(n)=C(n) .
$$

In [5], Alladi, Andrews, and Gordon obtained substantial refinements and generalizations of Theorem $\mathrm{G}$ by using a technique called the method of weighted words. We now describe briefly the main ideas in [5].

Theorem $\mathrm{G}$ is viewed in [5] as emerging out of the key identity

$$
\sum_{i, j, k} a^{i} b^{j} c^{k} \sum_{\substack{i=\alpha+\delta+\varepsilon \\ j=\beta+\delta+\phi \\ k=\gamma+\varepsilon+\phi}} \frac{q^{T_{s}+T_{\delta}+T_{\varepsilon}+T_{\phi-1}}\left(1-q^{\alpha}\left(1-q^{\phi}\right)\right)}{(q)_{\alpha}(q)_{\beta}(q)_{\gamma}(q)_{\delta}(q)_{\varepsilon}(q)_{\phi}}=(-a q)_{\infty}(-b q)_{\infty}(-c q)_{\infty}
$$

under the standard transformations

$$
\left.\begin{array}{c}
\text { (dilation) } q \mapsto q^{6}, \\
\text { (translations) } \quad a \mapsto a q^{-4}, b \mapsto b q^{-2}, c \mapsto c q^{-1} .
\end{array}\right\}
$$


In (5.1), $s=\alpha+\beta+\gamma+\delta+\varepsilon+\phi$, and $T_{m}=m(m+1) / 2$. Clearly when the transformations (5.2) are applied to the product in (5.1) we get

$$
\prod_{m=1}^{\infty}\left(1+a q^{6 m-4}\right)\left(1+b q^{6 m-2}\right)\left(1+c q^{6 m-1}\right)
$$

which is the three-parameter refined generating function of $B(n)$ in Theorem G. We now describe how the series in (5.1) becomes the refined generating function of $C(n)$ under the influence of $(5.2)$.

We consider the integer 1 as occurring in three primary colors $a, b$, and $c$, and integers $n \geq 2$ as occurring in the three primary colors as well as in three secondary colors $d=a b, e=a c$, and $f=b c$. The integer $n$ in color $a$ is denoted by the symbol $a_{n}$, with similar interpretation for the symbols $b_{n}, \ldots, f_{n}$. In order to discuss partitions (words) involving the symbols, we need an ordering among them, and the one we choose is

$$
a_{1}<b_{1}<c_{1}<d_{2}<e_{2}<a_{2}<f_{2}<b_{2}<c_{2}<d_{3}<e_{3}<a_{3}<f_{3}<\ldots
$$

The reason for choosing this ordering is because under the transformations (5.2) the symbols become

$$
\left.\begin{array}{c}
a_{n} \mapsto 6 n-4, b_{n} \mapsto 6 n-2, c_{n} \mapsto 6 n-1, \text { for } n \geq 1, \\
d_{n}=a b_{n} \mapsto 6 n-6, e_{n}=a c_{n} \mapsto 6 n-5, f_{n}=b c_{n} \mapsto 6 n-3, \text { for } n \geq 2,
\end{array}\right\}
$$

and so (5.4) becomes

$$
2<4<5<6<7<8<9<10<11<12<13<\ldots,
$$

the natural ordering among the integers.

It is convenient to write down the complete list of symbols in (5.4), namely,

$$
\underline{e}_{1}<a_{1}<\underline{f}_{1}<b_{1}<c_{1}<d_{2}<e_{2}<a_{2}<f_{2}<b_{2}<c_{2}<d_{3}<\ldots
$$

where $\underline{e}_{1}$ and $\underline{f}_{1}$ in (5.7) are underlined because they do not really occur. We have omitted writing $\underline{d}_{1}$ in (5.7) because the standard evaluation of $\underline{d}_{1}$ yields 0 .

Next, let $x_{n}$ denote the symbol occupying position $n$ in (5.7); that is, $x_{1}=$ $\underline{e}_{1}, x_{2}=a_{1}, x_{3}=\underline{f}_{1}$, and so on. By a Type- 1 partition we mean an expression of the form $x_{n_{1}}+x_{n_{2}}+\cdots+x_{n_{\nu}}$, where the $x_{n_{i}}$ are chosen from the non-underlined set in (5.7) and satisfy the standard gap conditions

$$
n_{i}-n_{i+1} \geq 6 \text {, with strict inequality if } x_{n_{i}} \text { is of secondary color. }
$$

The main result in [5] is

Theorem C. Let $V(n ; i, j, k)$ denote the number of vector partitions $\left(\pi_{1}^{\prime}, \pi_{2}^{\prime}, \pi_{3}^{\prime}\right)$ of $n$ such that $\pi_{1}^{\prime}$ has $i$ distinct parts all in color $a, \pi_{2}^{\prime}$ had $j$ distinct parts all in color $b$, and $\pi_{3}^{\prime}$ has $k$ distinct parts all in color $c$.

Let $C(n ; \alpha, \beta, \gamma, \delta, \varepsilon, \phi)$ denote the number of Type- 1 partitions of $n$ having $\alpha$ $a$-parts, $\beta$ b-parts, $\ldots, \phi f$-parts. Then

$$
V(n ; i, j, k)=\sum_{\substack{i=\alpha+\delta+\varepsilon \\ j=\beta+\delta+\phi \\ k=\gamma+\varepsilon+\phi}} C(n ; \alpha, \beta, \gamma, \delta, \varepsilon, \phi) .
$$

Clearly the generating function of $V(n ; i, j, k)$ is

$$
\sum_{i, j, k, n} V(n ; i, j, k) a^{i} b^{i} c^{k} q^{n}=(-a q)_{\infty}(-b q)_{\infty}(-c q)_{\infty} .
$$


In [5] it is shown that for given $\alpha, \beta, \gamma, \delta, \varepsilon$, and $\phi$,

$$
\sum_{n} C(n ; \alpha, \beta, \gamma, \delta, \varepsilon, \phi) q^{n}=\frac{q^{T_{s}+T_{\delta}+T_{\varepsilon}+T_{\phi-1}}\left(1-q^{\alpha}\left(1-q^{\phi}\right)\right)}{(q)_{\alpha}(q)_{\beta}(q)_{\gamma}(q)_{\delta}(q)_{\varepsilon}(q)_{\phi}} .
$$

Thus Theorem 3 is a consequence of (5.9), (5.10) and the key identity (5.1).

In this approach, under the transformations (5.1), the primary colors $a, b, c$, correspond to the residues $2,4,5(\bmod 6)$ which determine the partition function $B(n)$ in Theorem $\mathrm{G}$. Thus the secondary colors are $2+4 \equiv 6(\bmod 6), 2+5 \equiv$ $7(\bmod 6)$, and $4+5 \equiv 9(\bmod 6)$ meaning that the residue classes $0,1,3(\bmod 6)$ represent secondary colors, but parts in these residue classes are all $\geq 6$ because $\underline{d}_{1}, \underline{e}_{1}, \underline{f}_{1}$ do not occur. This explains the condition $m_{\nu} \neq 1$ or 3 in defining $C(n)$ in Theorem G. Also the strict inequality $m_{i}-m_{i+1}>6$ when $m_{i} \equiv 0,1$, or $3(\bmod 6)$ is to be interpreted as the inequality being strict when $m_{i}$ is of secondary color. The difference conditions defining Type- 1 partitions translate to those defining $C(n)$ in Theorem $\mathrm{G}$ when the standard transformations (5.2) are applied. Thus Theorem $\mathrm{C}$ is a strong refinement and generalization of Theorem $\mathrm{G}$.

\section{Proof of Theorem 1}

Instead of the standard transformations (5.2), let us consider the effect of the quadratic transformations

$$
\left.\begin{array}{c}
\text { (dilation) } \quad q \mapsto q^{2}, \\
\text { (translations) } \quad a \mapsto a q^{-1}, b \mapsto b q^{-1}, c \mapsto c,
\end{array}\right\}
$$

on (5.1). Clearly (6.1) converts the product on the right in (5.1) to the product in (2.1), which is the generating function of partitions $\vec{\pi} \in \mathcal{V}$ in Theorem 1 .

The effect of (6.1) on the symbols is

$$
\left.\begin{array}{c}
a_{n} \mapsto 2 n-1, \quad b_{n} \mapsto 2 n-1, \quad c_{n} \mapsto 2 n, \quad \text { for } n \geq 1, \\
d_{n}=a b_{n} \mapsto 2 n-2, e_{n}=a c_{n} \mapsto 2 n-1, f_{n}=b c_{n} \mapsto 2 n-1, \text { for } n \geq 2 .
\end{array}\right\}
$$

Thus (5.4) becomes

$$
1_{a}<1_{b}<2_{c}<2_{a b}<3_{a c}<3_{a}<3_{b c}<3_{b}<4_{c}<4_{a b}<5_{a c}<5_{a}<\ldots,
$$

where we have reversed the convention by indicating the color with a subscript. In this case Type- 1 partitions are Rogers-Ramanujan partitions satisfying certain color conditions which determine the weight of such a partition. These conditions imply that when two integers in (6.3) differ by $>2$, then colors can be attached to either integer in all possible ways. That is, there is no interference or dependence here. What this means is that in order to determine the weight of a Rogers-Ramanujan partition, we need only consider chains of parts and calculate the weights of these chains; then by the independence, the weight of the partition can be calculated using the product formula (1.2).

Consider now a chain $\chi$ of even parts $m_{1}>m_{2}>\cdots>m_{\ell}$. Note that (5.8) implies that we have a choice only for $m_{1}$ to have color $c$ or $a b$, but all parts $<m_{1}$ in the chain must have color $c$. Thus in this case

$$
\omega(\chi)=c^{\ell-1}(c+a b), \text { if } \lambda(\chi) \text { is even, }
$$

as in (1.1).

Next consider a chain $\chi$ with $\lambda(\chi)=1$. If any part in this chain has color $b$, then the next higher part (and therefore all parts higher) must have color $b$. If any 
part in this chain has color $b c$, then all parts higher must have color $b$. If any part in this chain has color $a$, then the next higher part can have color $a, b c$, or $b$. Note that no part in this chain can have color $a c$ since the chain has to start with colors $a$ or $b$. So we have the following cases to consider.

Case 1: 1 has color $b$.

Then all parts have color $b$. So the weight of this chain is $b^{l}$.

Case 2: All parts have color $a$.

Then clearly the weight of the chain is $a^{l}$.

Case 3: The only parts in color $a$ are $1,3, \ldots, 3 k-1$, with $1 \leq k<\ell=\ell(\chi)$.

Then $2 k+1$ can have color $b c$ or $b$, but all parts $>2 k+1$ must have color $b$. So the weight of this chain is

$$
a^{k}(b c+b) b^{\ell-k-1}=(1+c) a^{k} b^{\ell-k} .
$$

We need to sum this over all $k$ to get the weight of all chains covered by Case 3 .

The sum of the weights of chains in Cases 1, 2, and 3 is

$$
b^{\ell}+a^{\ell}+(1+c) \sum_{k=1}^{\ell-1} a^{k} b^{\ell-k}
$$

as in (1.1).

Finally, consider chains $\chi$ with $\lambda(\chi)=$ odd $>1$. In this situation we have color choices as in Cases 1, 2, 3 above, plus the cases where $\lambda(\chi)$ has color $a c$ or $b c$. So this gives rise to three more cases.

Case 4: $\lambda(\chi)$ has color $a c$, and the next part has color $b c$.

Then the rest of the parts have color $b$. So the weight of this chain is

$$
(a c)(b c) b^{\ell-2}=c^{2} a b^{\ell-1} \text {. }
$$

Case 5: $\lambda(\chi)$ has color $a c$, and the next part has color $a$ or $b$.

So the chain after $\lambda(\chi)$ has length $\ell-1$ and this situation covered by the colorings as in Cases 1, 2, and 3. So the weight in this case is

$$
\begin{aligned}
& a c\left\{a^{\ell-1}+(1+c)\left(a^{\ell-2} b+a^{\ell-3} b^{2}+\cdots+a b^{\ell-2}\right)+b^{\ell-1}\right\} \\
& =a^{\ell} c+c(1+c)\left(a^{\ell-1} b+a^{\ell-2} b^{2}+\cdots+a^{2} b^{\ell-2}\right)+c a b^{\ell-1} .
\end{aligned}
$$

Case 6: $\lambda(\chi)$ has color $b c$.

Then the rest of the parts all have color $b$. So the weight of this chain is

$$
(b c) b^{\ell-1}=c b^{\ell} \text {. }
$$

So adding the weights in (6.6), (6.7) and (6.8) we get

$$
c a^{\ell}+c(1+c)\left(a^{\ell-1} b+a^{\ell-2} b^{2}+\cdots+a b^{\ell-1}\right)+c b^{\ell} .
$$

Finally, we need to add the weights in (6.9) and (6.5) to get the weights of chains in Cases 1 through 6 that cover all chains with $\lambda(\chi)=$ odd $>1$. This gives

$$
(1+c) a^{\ell}+(1+c)^{2}\left(a^{\ell-1} b+a^{\ell-2} b^{2}+\cdots+a b^{\ell-1}\right)+(1+c) b^{\ell},
$$

which is what is given in (1.1).

Thus the weights in (1.1) have been established and this completes the proof of Theorem 1. 
Remarks. Previously we had discussed consequences of (5.1) and Theorem 1 under the dilations $q \mapsto q^{3}$ (see [1]) and $q \mapsto q^{4}$ (see [4]), and certain sets of translations. These cubic and quartic transformations lead to different combinatorial versions of Theorem G.

\section{SuCCESSIVE RANKS WITH PRESCRIBED BOUNDS}

The Ferrers graph of every partition contains a Durfee square, namely, the largest square of nodes starting from the top left-hand corner of the graph. Through every node on the descending diagonal of the Durfee square there is a hook passing through it, namely, the set of nodes from that node on the diagonal together with those that lie horizontally to its right and vertically below it. The Ferrers graph of the partition $7+6+6+4+4+2+1+1$, its Durfee square, and its hooks, are illustrated in Figure 1.

If the graph of a partition has a $k \times k$ Durfee square, then the number of hooks is $k$. The largest hook is called the first hook, the second largest hook is called the second hook, and so on.
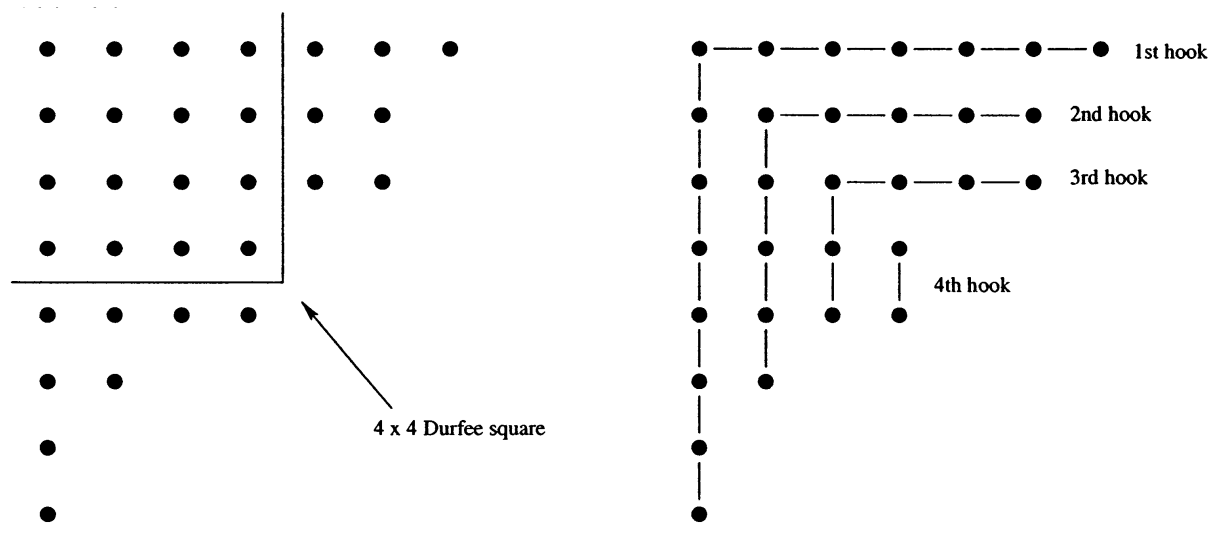

FIGURE 1.

Given a hook of a Ferrers graph, its rank is the number of horizontal nodes to the right of the vertex of the hook, minus the number of vertical nodes below the vertex of the hook. The rank of the $i$-th hook is called the $i$-th rank of the partition. The successive ranks in Figure 1 are $-1,0,1$, and -1 .

The first rank is, of course, the familiar rank of the partition made famous by Dyson 14 in conjecturing a combinatorial explanation of Ramanujan's congruences modulo 5 and 7 for the partition function. The Dyson conjectures involving the rank were proved by Atkin and Swinnerton-Dyer [12]. Atkin [11] was led to consider successive ranks while attempting a study of Ramanujan's partition congruence mod 11. Following Atkin, Andrews [8] and Bressoud [13] studied partitions with prescribed bounds for successive ranks. In [10] the notion of successive ranks was generalized to hook differences, with the hook vertices not necessarily on the diagonal of the Durfee square. Also in [10] the succesive rank theorem of Andrews 8. and Bressoud [13] was revised as follows:

Theorem R. Let $Q_{k, i}(n)$ denote the number of partitions of $n$ such that the successive ranks all take values in the interval $[-i+2, k-i-2]$. 
Let $A_{k, i}(n)$ denote the number of partitions into parts $\not \equiv 0, \pm i(\bmod k)$.

Then for $1 \leq i<k / 2$ we have

$$
Q_{k, i}(n)=A_{k, i}(n) .
$$

Although the case $2 i=k$ is not covered by Theorem $\mathrm{R}$, it is possible to deal with this case by defining $A_{k, i}(n)$ via the identity

$$
\sum_{n=0}^{\infty} A_{k, i}(n) q^{n}=\frac{\left(q^{k} ; q^{k}\right)_{\infty}\left(q^{i} ; q^{k}\right)_{\infty}\left(q^{k-i} ; q^{k}\right)_{\infty}}{(q)_{\infty}}
$$

If $2 i \neq k$, then $A_{k, i}(n)$ defined in (7.1) has the partition interpretation as in Theorem R. However, if $2 i=k$, then $A_{k, i}(n)$ does not have a partition interpretation in the standard sense. The advantage of (7.1) is that it leads to

\section{Theorem $\mathbf{R}^{\prime}$.}

$$
Q_{2 k, k}(n)=A_{2 k, k}(n)
$$

Remarks. Theorem $\mathrm{R}^{\prime}$ is a consequence of Theorem 5 of [10], but we stress here that if $A_{2 k, k}(n)$ is to be interpreted as the number of partitions of $n$ into parts $\not \equiv 0, \pm k(\bmod 2 k)$, then this has to be in the sense of $(7.1)$, where the residue class $k(\bmod 2 k)$ is "deleted twice" because it occurs as both $k$ and $-k(\bmod 2 k)$.

\section{A Weighted Partition theorem Mod 6}

Given a partition $\pi$ whose Ferrers graph has a $k \times k$ Durfee square, consider the partition $\rho(\pi)=\tilde{\pi}$ into $k$ parts $h_{1}+h_{2}+\ldots+h_{k}$, where $h_{i}$ is the number of nodes in (= the length of) the $i$-th hook of $\pi$. Note that $h_{i}-h_{i+1} \geq 2$ for $1 \leq i \leq k-1$ and so $\tilde{\pi}$ is a Rogers-Ramanujan partition. The mapping

$$
\pi \rightarrow \rho(\pi)=\tilde{\pi}
$$

is surjective. Also, if $r_{i}(\pi)$ is the $i$-th rank of $\pi$, then under this mapping

$$
h_{i}-r_{i}(\pi) \equiv 1(\bmod 2) .
$$

The study of the surjection (8.1) along with Theorems $\mathrm{R}$ and $\mathrm{R}^{\prime}$ will lead us to several weighted partition identities in this and subsequent sections. We begin with

Theorem 2. Given $\tilde{\pi} \in \mathcal{R}_{2}$, let its weight $\omega_{2}(\tilde{\pi})=2^{r}$, where $r$ is the number of even chains $\chi$ in $\tilde{\pi}$ with $\lambda(\chi)>2$.

Let $A_{6,1}(n)$ denote the number of partitions of $n$ into parts $\not \equiv 0, \pm 1(\bmod 6)$. Then

$$
A_{6,1}(n)=\sum_{\tilde{\pi} \in \mathcal{R}_{2}, \sigma(\tilde{\pi})=n} \omega_{2}(\tilde{\pi}) .
$$

Proof. Take $k=6, i=1$ in Theorem R. Thus

$$
A_{6,1}(n)=Q_{6,1}(n)
$$

We will show that

$$
Q_{6,1}(n)=\sum_{\tilde{\pi} \in \mathcal{R}_{2}, \sigma(\tilde{\pi})=n} \omega_{2}(\tilde{\pi}) .
$$

Theorem 2 will follow from (8.3) and (8.4). 
We know that $Q_{6,1}(n)$ is the number of partitions of $n$ whose successive ranks take values 1,2 , or 3 . Given a partition $\pi$ enumerated by $Q_{6,1}(n)$, consider the partition $\rho(\pi)=\tilde{\pi}$ generated by the hooks of $\pi$. If the $i$-th part of $\tilde{\pi}$ ( $=i$-th hook length of $\pi$ ) is odd, then by (8.2) the $i$-th rank of $\pi$ must be even, and so must be 2. So there is only one way in which this hook can occur. For instance, if 7 is the hook length and the rank is 2, then the hook must be as shown in Figure 2.

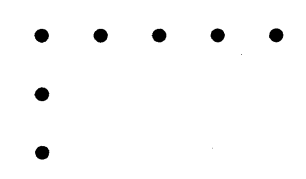

FIGURE 2.

Note that 1 can never occur as a hook length with rank 2. Thus 1 cannot occur as a part of $\tilde{\pi}$. Hence $\tilde{\pi} \in \mathcal{R}_{2}$.

Now if the $i$-th part of $\tilde{\pi}$ is even, then the $i$-th rank of $\pi$ could be either 1 or 3 . The question is under what circumstances can both values 1 and 3 occur as the rank?

The integer 2 as a hook length can occur only with rank 1 and cannot have rank 3. But even integers $>2$ can arise as hook lengths in two ways, one with rank 1 , and another with rank 3. For instance, 6 as a hook length can be realized as shown in Figure 3.

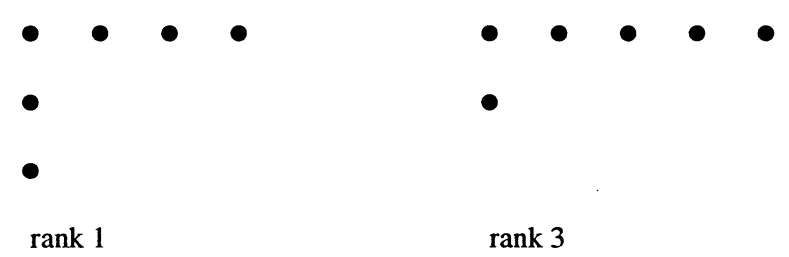

FIGURE 3.

If two consecutive integers $2 j$ and $2 j+2$ occur as parts of $\tilde{\pi}$, and if the hook representing $2 j$ has a certain rank, then the hook representing $2 j+2$ must have the same rank. On the other hand, if two consecutive parts of $\pi$ differ by $>2$, then the rank of one part is independent of the rank of the other. Because of this independence, the weight to be attached to $\tilde{\pi}$ can be computed by decomposing $\tilde{\pi}$ into chains and taking the product of the weights of chains.

So given $\tilde{\pi} \in \mathcal{R}_{2}$, decompose it into chains. All odd parts of $\tilde{\pi}$ have unique representations as hook lengths of $\pi$. With regard to even chains $\chi$ of $\tilde{\pi}$, the hook representation of $\lambda(\chi)$ in $\pi$ uniquely determines the hook representation of all other parts in that chain. If $\lambda(\chi)>2$ is even, then $\lambda(\chi)$ admits two representations as a hook, one with rank 1 and another with rank 3 . If $\lambda(\chi)=2$, then the hook representation must have rank 1 . Thus to each $\tilde{\pi} \in \mathcal{R}_{2}$ there correspond $2^{r}$ partitions $\pi$ enumerated by $Q_{6,1}$, such that $\rho(\pi)=\tilde{\pi}$. This establishes (8.4) and completes the proof of Theorem 2. 


\section{TWO MORE WeIGHTED IDENTITIES MOD 6}

Pursuing the ideas of 8 , we get two more results.

Theorem 3. Given a Rogers-Ramanujan partition $\tilde{\pi}$, let $\omega_{3}(\tilde{\pi})=2^{r}$, where $r$ is the number of odd chains $\chi$ of $\tilde{\pi}$ with $\lambda(\chi)>1$.

Let $A_{6,2}(n)$ denote the number of partitions of $n$ into parts $\not \equiv 0, \pm 2(\bmod 6)$. Then

$$
A_{6,2}(n)=\sum_{\tilde{\pi} \in \mathcal{R}, \sigma(\tilde{\pi})=n} \omega_{3}(\tilde{\pi}) .
$$

Proof. We know from Theorem $\mathrm{R}$ that

$$
A_{6,2}(n)=Q_{6,2}(n)
$$

Recall that $Q_{6,2}(n)$ is the number of partitions $\pi$ of $n$ such that the successive ranks take values 0,1 , or 2 .

Given $\tilde{\pi} \in \mathcal{R}$, consider a partition $\pi$ enumerated by $Q_{6,2}$ with $\rho(\pi)=\tilde{\pi}$. In this case, the even parts of $\tilde{\pi}$ have unique representations as hooks of $\pi$ because the rank must be 1 . The odd parts of $\tilde{\pi}$ which are $>1$ can have two possible representations as hooks of $\pi$-one with rank 0 and another with rank 2 . In a chain $\chi$ of odd parts of $\tilde{\pi}$, the hook representation of $\lambda(\chi)$ uniquely represents the hook representation of all other parts in the chain. When $\lambda(\chi)>1$ is odd, it admits two hook representations in $\pi$, but $\lambda(\chi)=1$ admits only one representation with rank 0 . Thus to each $\tilde{\pi} \in \mathcal{R}$, there correspond $\omega_{3}(\tilde{\pi})$ partitions $\pi$ enumerated by $Q_{6,2}$ such that $\rho(\pi)=\tilde{\pi}$. Therefore

$$
Q_{6,2}(n)=\sum_{\tilde{\pi} \in \mathcal{R}, \sigma(\tilde{\pi})=n} \omega_{3}(\tilde{\pi}) .
$$

Theorem 3 follows from (9.1) and (9.2).

Remarks. Note that $A_{6,2}(n)$ is the number of partitions of $n$ into odd parts because

$$
\sum_{n=0}^{\infty} Q_{6,2}(n) q^{n}=\frac{1}{\left(q ; q^{6}\right)_{\infty}\left(q^{3} ; q^{6}\right)_{\infty}\left(q^{5} ; q^{6}\right)_{\infty}}=\frac{1}{\left(q ; q^{2}\right)_{\infty}}
$$

By Euler's theorem, $Q_{6,2}(n)$ equals $D(n)$, the number of partitions of $n$ into distinct parts. Thus Theorem 3 is a reformulation of Theorem A but here the result is proved using successive ranks. In doing so, we see a similarity with Theorem 2.

We now consider the one remaining product mod 6 , namely,

$$
\begin{aligned}
& \sum_{n=0}^{\infty} A_{6,3}(n) q^{n}=\frac{\left(q^{6} ; q^{6}\right)_{\infty}\left(q^{3} ; q^{6}\right)_{\infty}\left(q^{3} ; q^{6}\right)_{\infty}}{(q)_{\infty}} \\
= & \frac{\left(q^{3} ; q^{6}\right)_{\infty}}{\left(q ; q^{3}\right)_{\infty}\left(q^{2} ; q^{3}\right)_{\infty}}=\frac{1}{\left(q ; q^{3}\right)_{\infty}\left(q^{2} ; q^{3}\right)_{\infty}\left(-q^{3} ; q^{3}\right)_{\infty}} .
\end{aligned}
$$

The product on the right in (9.4) is the generating function for unrestricted partitions $\pi$ which are counted with weight $(-1)^{\nu_{3}(\pi)}$, with $\nu_{3}(\pi)$ denoting the number of multiples of 3 in $\pi$.

Next, $Q_{6,3}(n)$ enumerates partitions $\pi$ of $n$ whose successive ranks take values -1 , 0 , or 1 . By considering partitions $\tilde{\pi} \in \mathcal{R}$ with $\tilde{\pi}=\rho(\pi)$, and by following the 
reasoning in $\S 8$, we see that to each $\tilde{\pi}$ there correspond $\omega_{4}(\pi)=2^{r}$ partitions $\pi$ enumerated by $Q_{6,3}$, where $r$ is the number of even chains of $\tilde{\pi}$. Thus

$$
Q_{6,3}(n)=\sum_{\tilde{\pi} \in \mathcal{R}, \sigma(\tilde{\pi})=n} \omega_{4}(\tilde{\pi}) .
$$

By Theorem $\mathrm{R}^{\prime}$ we know that

$$
A_{6,3}(n)=Q_{6,3}(n)
$$

So from (9.4), (9.5), and (9.6) we get

Theorem 4. Let $\mathcal{U}$ denote the set of all (unrestricted) partitions. Then

$$
\sum_{\pi \in \mathcal{U}, \sigma(\pi)=n}(-1)^{\nu_{3}(\pi)}=\sum_{\tilde{\pi} \in \mathcal{R}, \sigma(\tilde{\pi})=n} \omega_{4}(\tilde{\pi}) .
$$

\section{A Weighted PARTition TheOREM Mod 7}

In this and the next section we will establish three results connecting weighted Rogers-Ramanujan partitions and partitions into parts $\not \equiv 0 \pm i(\bmod 7)$, for $i=$ $1,2,3$. The weights in all three cases turn out to be products of Fibonacci numbers, which are defined by

$$
F_{0}=0, \quad F_{1}=1, \quad F_{n}=F_{n-1}+F_{n-2}, \quad \text { for } \quad n \geq 2 .
$$

The Fibonacci numbers enter into the discussion naturally owing to the following lemma, which can be easily proved by induction (see [17, Sec. 7.1):

Lemma. Suppose there are $n$ boxes arranged in a certain order, and that each box can either be empty or filled. Then the number of ways in which no two consecutive boxes can both be empty is $F_{n+2}$.

Next, we define a string $\psi$ in a Rogers-Ramanujan partition $\mathcal{R}$ to be a maximal sequence of parts where the difference between consecutive parts is $\leq 3$. Thus two strings are separated by gap $\geq 4$, and every Rogers-Ramanujan partition can be decomposed into strings.

Given a string $\psi$, let $\eta(\psi)$ denote the number of gaps equal to 3 in $\psi$. The weight $\omega_{5}(\psi)$ of $\psi$ is defined as

$$
\omega_{5}(\psi)= \begin{cases}F_{\eta+3}, & \text { if } \quad 1 \notin \psi, \\ F_{\eta+2}, & \text { if } \quad 1 \in \psi .\end{cases}
$$

The weight of a Rogers-Ramanujan partition $\tilde{\pi}$ is defined multiplicatively as

$$
\omega_{5}(\tilde{\pi})=\prod_{\psi} \omega_{5}(\psi)
$$

where the product is taken over all strings $\psi$ in $\tilde{\pi}$.

We are now in a position to state our first mod 7 theorem.

Theorem 5. Let $A_{7,3}(n)$ denote the number of partitions of $n$ into parts $\not \equiv 0, \pm 3$ $(\bmod 7)$. Then

$$
A_{7,3}(n)=\sum_{\tilde{\pi} \in \mathcal{R}, \sigma(\tilde{\pi})=n} \omega_{5}(\tilde{\pi}) .
$$


Proof. We know from Theorem $\mathrm{R}$ that

$$
A_{7,3}(n)=Q_{7,3}(n),
$$

where $Q_{7,3}(n)$ is the number of partitions $\pi$ of $n$ such that the successive ranks take values $-1,0,1$, or 2 . We will show that

$$
Q_{7,3}(n)=\sum_{\tilde{\pi} \in \mathcal{R}, \sigma(\tilde{\pi})=n,} \omega_{5}(\tilde{\pi}) .
$$

Theorem 5 will follow from (10.4) and (10.5).

Consider all partitions $\pi$ enumerated by $Q_{7,3}(n)$ and the partitions $\rho(\pi)=\tilde{\pi}$ they generate. Any even part of $\tilde{\pi}$ can occur as a hook length of a certain $\pi$ with rank either -1 or 1 . Any odd part $>1$ of $\tilde{\pi}$ can occur as a hook length of a certain $\pi$ with rank 0 or 2 , but 1 as a part can only occur as a hook with rank 0 .

If two parts $j$ and $j+2$ of $\tilde{\pi}$ differ by 2 , then the hook representation of $j$ uniquely determines the hook representation of $j+2$ because both hooks must have the same rank. If two consecutive parts of $\tilde{\pi}$ differ by $\geq 4$, then the hook representation of one has no influence on the hook representation of the other in $\pi$. This explains why the multiplicative formula (10.3) is true. So we need only determine the weights of strings $\psi$ and for this purpose we concentrate on gaps in $\psi$ which are exactly 3 .

If two consecutive parts of $\tilde{\pi}$ differ by 3 , then in their hook representations, the only disallowed rank combinations are -1 and 2 , or 2 and -1 , depending on whether the larger part is even or odd. For instance, if 6 and 9 are consecutive parts of $\tilde{\pi}$, then the allowable hook representations are shown in Figure 4.

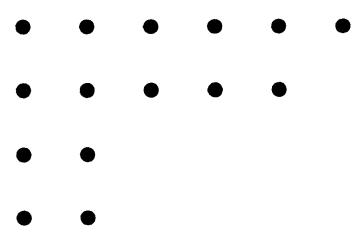

ranks: 2,1

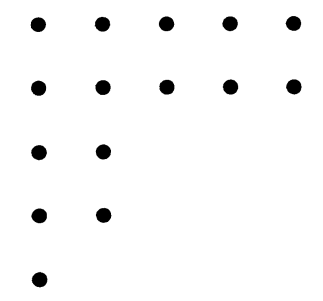

ranks: 0,1

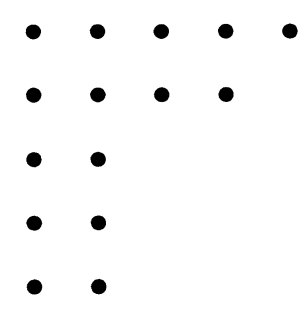

ranks: $0,-1$

\section{FiguRE 4.}

When two parts of a string differ by 3 , they are of opposite parity. If two parts of a string differ by 2 , then they are of the same parity and their hook representations have identical rank. Thus all hook representations of parts in a given chain will have the same rank once the rank of the hook representation of the smallest part of the chain is decided. It is the choice of assigning this rank to the smallest part of a chain, and consequently to the chain itself, that leads to weights. Thus for the purpose of determining the weights of strings, we may consider the decomposition of strings into chains.

Consider now a string $\psi$ all of whose parts are $>1$. Let $\psi$ have exactly $\eta$ gaps equal to 3 . This means there are $\eta+1$ chains that generate these $\eta$ gaps. One may think of these chains as numbered boxes. Adjacent chains correspond to boxes of opposite parity. If an odd chain in $\psi$ is represented by hooks all with rank 0 , one may think of the box corresponding to it as being non-empty; if all hooks in the 
odd chain have rank 2, one may think of the box as being empty. Similarly, if an even chain is represented by hooks all with rank 1 , one may think of the box as being non-empty, and if the hooks representing the even chain all have rank -1 , one may think of the corresponding box as being empty. We need to assign ranks to the hook representations of these chains such that ranks 2 and -1 , or -1 and 2 cannot occur as rank combinations of adjacent chains (of hooks). This is the same as saying that the $\eta+1$ boxes have to be filled in such a way that no two adjacent boxes can be empty. From the lemma we know that there are $F_{\eta+3}$ ways of doing this. Thus the weight attached to a string $\psi$ not having 1 as a part is $F_{\eta+3}$ as in (10.2).

Now if 1 is a part of $\psi$, then the chain in $\psi$ having 1 in it must have rank 0 for the hook representation of all its parts, leaving us no other choice. So if $\psi$ has $\eta$ gaps equal to 3 , we must ignore the gap of 3 between the chain having 1 as a part and the next chain in computing $\omega_{5}(\psi)$. Thus we consider only $\eta-1$ gaps of 3 and the $\eta$ chains that generate these gaps. By the reasoning of the preceding paragraph, the weight to be assigned to such a string will be $F_{\eta+2}$ as in (10.2). Thus the weights in (10.2) have been established.

From the multiplicative definition in (10.3) it follows that to each $\tilde{\pi} \in \mathcal{R}$, there correspond $\omega_{5}(\tilde{\pi})$ partitions $\pi$ enumerated by $Q_{7,3}$ such that $\rho(\pi)=\tilde{\pi}$. This establishes (10.5) and so Theorem 5 is proved.

\section{Two MORE WeIGHTEd IDENTITIES MOd 7}

The definition of weights of strings in the next two theorems will be a bit more complicated compared to (10.2) and the reasons will become clear soon. The proofs however will use methods identical to that of $\S 10$.

Given a Rogers-Ramanujan partition $\tilde{\pi}$, decompose it into strings $\psi$ as before. Suppose $\psi$ has $\eta$ gaps equal to 3 . Then the weight $\omega_{6}(\psi)$ is

$$
\omega_{6}(\psi)= \begin{cases}F_{\eta+3}, & \text { if all parts of } \psi \text { are } \geq 3, \\ F_{\eta+2}, & \text { if either } 1 \in \psi \text { and } 3 \notin \psi, 4 \notin \psi, \quad \text { or } 2 \in \psi, \\ F_{\eta+1}, & \text { if } 1 \in \psi \text { and either } 3 \text { or } 4 \in \psi .\end{cases}
$$

As before, the weight of $\omega_{6}(\tilde{\pi})$ is defined multiplicatively as

$$
\omega_{6}(\tilde{\pi})=\prod_{\psi} \omega_{6}(\psi)
$$

We then have

Theorem 6. Let $A_{7,2}(n)$ denote the number of partitions of $n$ into parts $\not \equiv 0, \pm 2$ $(\bmod 7)$. Then

$$
A_{7,2}(n)=\sum_{\tilde{\pi} \in \mathcal{R}, \sigma(\tilde{\pi})=n} \omega_{6}(\tilde{\pi}) .
$$

Proof. We know from Theorem $\mathrm{R}$ that

$$
A_{7,2}(n)=Q_{7,2}(n),
$$


where $Q_{7,2}(n)$ is the number of partitions $\pi$ of $n$ such that the successive ranks take values $0,1,2$, or 3 . We will show that

$$
Q_{7,2}(n)=\sum_{\tilde{\pi} \in \mathcal{R}, \sigma(\tilde{\pi})=n} \omega_{6}(\tilde{\pi}) .
$$

Theorem 6 will follow from (11.3) and (11.4).

We consider partitions $\pi$ enumerated by $Q_{7,2}(n)$ and the partitions $\rho(\pi)=\tilde{\pi}$ they generate. If two parts of $\tilde{\pi}$ differ by $\geq 4$, then the hook representation of one does not influence the hook representation of the other. Thus $\tilde{\pi}$ can be decomposed into strings and $\omega_{6}(\tilde{\pi})$ can be defined multiplicatively as in (11.2) because of this independence.

In order to compute $\omega_{6}(\psi)$ for a string $\psi$, we observe that odd integers $>1$ can be represented by hooks with rank 0 or 2 , and even integers $>2$ can be represented by hooks with rank 1 or 3 . The integer 1 has a unique representation as a hook with rank 0 and similarly 2 has a unique representation as a hook with rank 1 . If parts of $\psi$ differ by 3 , then in their hook representation, the disallowed rank combinations are 0 and 3 , or 3 and 0 , for adjacent hooks depending on whether the larger part is odd or even. (The main difference between Theorems 6 and 5 is that in Theorem 6 , the unique rank of 1 is 0 , and 0 is part of the disallowed combination, whereas in Theorem 5 , the unique rank of 1 was 0 , but the disallowed combination there did not have 1 in it.) This is the reason for the extra complication in the definition of weights in (11.1).

If all parts of $\psi$ are $>4$, then clearly $\omega_{6}(\psi)=F_{\eta+3}$ as before. However, if $1 \in \psi$ and either 3 or $4 \in \psi$, then 1 has unique rank 0 , and since 0,3 , is a disallowed rank combination, the even chain following the chain containing 1 , can only be assigned the rank 1 for its hook representation. Thus we need to discard the first two chains in computing weights, and so the number of chains to be considered is only $\eta-1$ instead of $\eta+1$. So by the lemma, the weight of $\psi$ will be $F_{\eta+1}$ as in (11.1).

If $1 \in \psi$ but neither 3 nor 4 are in $\psi$, then 1 is a string by itself and its weight is 1 . We can think of this as $F_{0+2}=F_{2}=1$ with $\eta=0$.

If $2 \in \psi$, then 2 admits a unique representation as a hook. This forces us to eliminate the chain containing 2 in computing weights and so we have $\eta$ chains to consider instead of $\eta+1$. So the weight in this case is $F_{\eta+2}$ as in (11.1). Thus we have established the weight formula (11.1). Therefore to each $\tilde{\pi} \in \mathcal{R}$, there correspond $\omega_{6}(\tilde{\pi})$ partitions $\pi$ enumerated by $Q_{7,2}$ such that $\rho(\pi)=\tilde{\pi}$. Thus (11.4) is established, and this in conjunction with (11.3) yields Theorem 6 .

To discuss the final weighted partition theorem $\bmod 7$, we consider the decomposition of $\tilde{\pi} \in \mathcal{R}_{2}$ into strings $\psi$. Let, as before, $\eta$ denote the number of gaps equal to 3 in $\psi$. The weights $\omega_{7}(\psi)$ are defined by

$$
\omega_{7}(\psi)= \begin{cases}F_{\eta+3}, & \text { if all parts of } \psi \text { are } \geq 4, \\ F_{\eta+2}, & \text { if either } 2 \in \psi \text { and } 4 \notin \psi, 5 \notin \psi, \quad \text { or } \quad 3 \in \psi, \\ F_{\eta+1}, & \text { if } 2 \in \psi \text { and either } 4 \text { or } 5 \in \psi .\end{cases}
$$

As always, the weight of $\omega_{7}(\tilde{\pi})$ is defined multiplicatively as

$$
\omega_{7}(\tilde{\pi})=\prod_{\psi} \omega_{7}(\psi)
$$

We then have 
Theorem 7. Let $A_{7,1}(n)$ denote the number of partitions of $n$ into parts $\not \equiv 0, \pm 1$ $(\bmod 7)$. Then

$$
A_{7,1}(n)=\sum_{\tilde{\pi} \in \mathcal{R}_{2}, \sigma(\tilde{\pi})=n} \omega_{7}(\tilde{\pi}) .
$$

Proof. We know from Theorem $\mathrm{R}$ that

$$
A_{7,1}(n)=Q_{7,1}(n),
$$

where $Q_{7,1}(n)$ is the number of partitions $\pi$ of $n$ such that the successive ranks take values $1,2,3$, or 4 . We will show that

$$
Q_{7,1}(n)=\sum_{\tilde{\pi} \in \mathcal{R}_{2}, \sigma(\tilde{\pi})=n} \omega_{7}(\tilde{\pi}) .
$$

Theorem 7 will follow from (11.7) and (11.8).

Consider partitions $\pi$ enumerated by $Q_{7,1}(n)$ and the partitions $\rho(\pi)=\tilde{\pi}$ they generate. As before, owing to independence in assigning hook representations for adjacent parts of $\tilde{\pi}$ differing by $\geq 4$, we get the multiplicative formula (11.6).

In order to determine the weights of strings, we observe that odd integers $>4$ admit hook representations with rank 2 or 4 , and all even integers $>2$ admit hook representations with rank 1 or 3 . The integer 2 has a unique representation as a hook with rank 1, and the integer 3 has a unique representation as a hook with rank 2 . The integer 1 can only have a hook representation with rank 0 , but 0 is not an allowed value of the rank. Thus all parts of $\tilde{\pi}$ are $>1$, and so $\tilde{\pi} \in \mathcal{R}_{2}$.

If all parts of $\psi$ are $\geq 4$, then we can consider all $\eta+1$ chains that generate the $\eta$ gaps, and the weight is $F_{\eta+3}$ as in (11.5).

The disallowed rank combinations for adjacent hook lengths differing by 3 are 1 and 4 or 4 and 1 . So if $2 \in \psi$ and either 4 or $5 \in \psi$, then the even chain containing 2 has rank 1 in its hook representation, and the odd chain following it must have rank 2 for its hook representation. So we can consider only $\eta-1$ chains in computing weights, and the weight in this case is $F_{\eta+1}$ as in (11.5). Now if $2 \in \psi$ and neither 4 nor 5 belong to $\psi$, then 2 is a string by itself with weight 1 , which is to be interpreted as $F_{0+2}=1$ with $\eta=0$ as in (11.5).

Finally, if $3 \in \psi$, then the chain containing 3 has rank 2 for its hook representation and must be discarded in computing weights. So we have $\eta$ chains to consider, and the weight of $\psi$ by the lemma is $F_{\eta+2}$ as in (11.5).

Therefore to each $\tilde{\pi} \in \mathcal{R}_{2}$, there correspond $\omega_{7}(\tilde{\pi})$ partitions $\pi$ enumerated by $Q_{7,1}$ such that $\rho(\pi)=\tilde{\pi}$. Thus (11.8) is established, and this in conjunction with (11.7) yields Theorem 7 .

Remarks. In [3], weights that are products of Fibonacci numbers are attached to partitions into parts differing by $\geq 4$ and these lead to Rogers-Ramanujan partitions. Here we are attaching such weights to the Rogers-Ramanujan partitions and showing that these lead to partitions into parts $\not \equiv 0, \pm i(\bmod 7)$, for $i=1,2,3$. The first time partitions into parts $\not \equiv 0, \pm i(\bmod 7)$ were discussed in the context of extensions of the Rogers-Ramanujan partition theorems was by Gordon [16]. In this paper only the congruential side mod 7 in Gordon's theorems is considered and not his difference conditions. 


\section{Prospects}

Recently we have obtained a bounded version of the Göllnitz partition theorem (see Theorem 1 of [6]), which is a stronger form of Theorem $\mathrm{C}$ of $\S 5$, where in addition to the conditions of Theorem $\mathrm{C}$, certain upper bounds are imposed on the parts. If these upper bounds tend to $\infty$, that is, if the bounds on the sizes of the parts are removed, then Theorem 1 in [6] reduces to Theorem C. This stronger result is deduced as a consequence of a new finite identity which reduces to (5.1) when the bounds tend to infinity. By applying the method of $\S 6$ to this bounded version of the Göllnitz theorem, we are able to obtain the following new finite versions of the Jacobi triple product identity:

$$
\begin{gathered}
\sum_{\ell=0}^{L}(-1)^{L+\ell} q^{2\left(T_{L}-T_{\ell}\right)} \sum_{n=-\ell}^{\ell} A^{n} q^{n^{2}} \\
=\sum_{i, j, k}(-1)^{k} A^{i-j} q^{2 T_{i}+2 T_{j}+2 T_{k}-i-j}\left[\begin{array}{c}
L-k \\
i
\end{array}\right]_{q^{2}}\left[\begin{array}{c}
L-i \\
j
\end{array}\right]_{q^{2}}\left[\begin{array}{c}
L-j \\
k
\end{array}\right]_{q^{2}},
\end{gathered}
$$

and Lebesgue's identity

$$
\begin{gathered}
\sum_{r, s} q^{2\left(T_{r}+T_{s}\right)}\left[\begin{array}{c}
L-s \\
r
\end{array}\right]_{q^{2}}\left[\begin{array}{c}
r+1 \\
s
\end{array}\right]_{q^{2}}(-1)^{s} A^{2 s} \\
=\sum_{i, j, k}(-1)^{j} q^{2\left(T_{i}+T_{j}+T_{k}\right)-i-j} A^{i+j}\left[\begin{array}{c}
L+1-k \\
i
\end{array}\right]_{q^{2}}\left[\begin{array}{c}
L+1-i \\
j
\end{array}\right]_{q^{2}}\left[\begin{array}{c}
L+1-j \\
k
\end{array}\right]_{q^{2}} \\
-q^{2(L+1)} \sum_{i, j, k}(-1)^{j} q^{2\left(T_{i}+T_{j}+T_{k}\right)-i-j} A^{i+j}\left[\begin{array}{c}
L-k \\
i
\end{array}\right]_{q^{2}}\left[\begin{array}{c}
L-i \\
j
\end{array}\right]_{q^{2}}\left[\begin{array}{c}
L-j \\
k
\end{array}\right]_{q^{2}} .
\end{gathered}
$$

In (12.1) and (12.2) the symbols $\left[\begin{array}{l}n \\ m\end{array}\right]_{q}$ are defined by

$$
\left[\begin{array}{c}
n \\
m
\end{array}\right]_{q}=\frac{(q)_{n}}{(q)_{m}(q)_{n-m}}
$$

for integers $n \geq m \geq 0$. When $L$ tends to infinity, (12.1) reduces to (2.7), and (12.2) essentially reduces to Lebesgue's identity dilated by a factor of 2 (see Andrews [9], Ch. 2)

$$
\left(1-A q^{2}\right) \sum_{r} \frac{q^{2 T_{r}}\left(A^{2} q^{4} ; q^{2}\right)_{r}}{\left(q^{2} ; q^{2}\right)_{r}}=\left(-q^{2} ; q^{2}\right)_{\infty}\left(A^{2} q^{2} ; q^{4}\right)_{\infty}=\frac{\left(A^{2} q^{2} ; q^{4}\right)_{\infty}}{\left(q^{2} ; q^{4}\right)_{\infty}}
$$

from which Theorem S follows. In deriving (12.1) and (12.2), special attention must be paid to the ordering (6.3). This is because the bounds on the parts in Theorem A lead to certain exceptional cases at the boundary when the transformations (6.2) are applied (see [7] for details).

In the second part of this paper we have concentrated on hooks that have vertices on the main diagonal of the Durfee square, and hook differences (successive ranks) 
that take either 3 or 4 consecutive integer values. When the number of values taken by the rank is 3 , the weights turned out to be powers of 2 as in Sections 8 and 9. When the successive ranks took four consecutive integer values as in Sections 10 and 11, the weights were products of Fibonacci numbers. One way to generalize this is to consider successive ranks taking more integer values and discuss the weighted identities they lead to. With this in mind, we have recently investigated the case of 5 successive integer values for the ranks, and even here the weights are more intricate than the ones considered in this paper. In this situation we need to decompose partitions in $\mathcal{R}$ into blocks of parts differing by $\leq 4$. If such a block has all gaps $\leq 3$, then its weight is of the form a power of 2 times a power of 3 , but otherwise the determination of the weights is more involved.

An even more challenging question is to consider partitions with prescribed hook differences where the hook vertices are not on the main diagonal as in [10], and discuss the weighted partition theorems they lead to. We plan to consider such questions in the future.

\section{ACKNOWLEDGEMENTS}

We would like to thank Frank Garvan for help with the diagrams and the referee for his comments and suggestions.

\section{REFERENCES}

1. K. Alladi, A combinatorial correspondence related to Göllnitz's (big) partition theorem and applications, Trans. Amer. Math. Soc. 349 (1997), 2721-2735. MR 97i:11106

2. K. Alladi, Partition identities involving gaps and weights, Trans. Amer. Math. Soc. 349 (1997), 5001-5019. MR 98c:05012

3. K. Alladi, Partition identities involving gaps and weights II, The Ramanujan J. 2 (1998), 21-37. MR 2000a:11149

4. K. Alladi, On a partition theorem of Göllnitz and quartic transformations (with an appendix by B. Gordon), J. Number Theory 69 (1998), 153-180. MR 99d:11112

5. K. Alladi, G. E. Andrews, and B. Gordon, Generalizations and refinements of a partition theorem of Göllnitz, Jour. Reine Angew. Math. 460 (1995), 165-188. MR 96c:11119

6. K. Alladi and A. Berkovich, A double bounded key identity for Göllnitz's (Big) partition theorem, in Symbolic Computation, Number Theory, Special Functions, Physics, and Combinatorics, Frank Garvan and Mourad Ismail, Eds., Developments in Mathematics Vol. 4, Kluwer Academic Publishers, Dordrecht (2001), 13-32.(see also CO/0007001)

7. K. Alladi and A. Berkovich, New finite versions of Jacobi's triple product, Sylvester, and Lebesgue identities (in preparation).

8. G. E. Andrews, Sieves in the theory of partitions, Amer. J. Math. 94 (1972), 1214-1230. MR 47:8424

9. G. E. Andrews, The theory of partitions, Encyclopedia of Math. and its Appl., Vol. 2, Addison Wesley, Reading, MA (1976). MR 58:27738

10. G. E. Andrews, R. J. Baxter, D. M. Bressoud, W. H. Burge, P. J. Forrester, and G. Viennot, Partitions with prescribed hook differences, European J. Combin. 8 (1987), 341-350. MR 89g:05014

11. A. O. L. Atkin, A note on ranks and the conjugacy of partitions, Quart. J. Math., Oxford (2) 17 (1966), 355-358. MR 34:2548

12. A. O. L. Atkin and H. P. F. Swinnerton-Dyer, Some properties of partitions, Proc. London Math. Soc. (3) 4 (1954), 84-106. MR 15:685d

13. D. M. Bressoud, Extension of the partition sieve, J. Number Theory 12 (1980), 87-100. MR 82e: 10022

14. F. J. Dyson, Some guesses in the theory of partitions, Eureka 8 (1944), 10-15.

15. H. Göllnitz, Partitionen mit Differenzenbedingungen, Jour. Reine Angew. Math. 225 (1967), 154-190. MR 35:2848 
16. B. Gordon, A combinatorial generalization of the Rogers-Ramanujan identities, Amer. J. Math. 83 (1961), 393-399. MR 23:A809

17. R. L. Graham, D. E. Knuth, and O. Patashnik, Concrete Mathematics, Addison Wesley, Reading, Massachusetts (1994). MR 97d:68003

18. J. J. Sylvester, A constructive theory of partitions in three Acts-an Interact, and an Exodium, Amer. J. Math. 5 (1882), 251-330.

Department of Mathematics, University of Florida, Gainesville, Florida 32611

E-mail address: alladi@math.ufl.edu

Department of Mathematics, University of Florida, Gainesville, Florida 32611

E-mail address: alexb@math.ufl.edu 